\title{
Preliminary Results using Timepix as a Particle Tracking Detector
}

\section{R. Plackett ${ }^{* a}$ K. Akiba, ${ }^{a}$ M. Artuso, ${ }^{b}$ F. Bayer, ${ }^{c}$ J. Buytaert ${ }^{a}$, M. Campbell, ${ }^{a}$ P. Collins, ${ }^{a}$ M. Crossley, ${ }^{a}$ R. Dumps, ${ }^{a}$ L. Eklund, ${ }^{d}$, D. Esperante ${ }^{e}$, L. Ferre Llin, ${ }^{d}$ A. Gallas, ${ }^{e}$ M. Gandelman, ${ }^{a}$ M. Gersabeck, ${ }^{d}$ V. Gligorov, ${ }^{a d}$ T. Huse, ${ }^{f}$ M. John, ${ }^{g}$ X. Llopart, ${ }^{a}$ D. Maneuski, ${ }^{d}$ T. Michel,${ }^{c}$ M. Nicol, ${ }^{a}$ C. Parkes, ${ }^{d}$ T. Poikela, ${ }^{a}$ E. Rodrigues, ${ }^{d}$ L. Tlustos ${ }^{a}$}

${ }^{a}$ CERN, European Organisation of Nuclear Research, Geneva, Switzerland.

${ }^{b}$ Syracuse University, Syracuse, NY, USA.

${ }^{c}$ Friedrich-Alexander-Universität Erlangen-Nürnberg, Franconia, Germany.

d University of Glasgow, Glasgow, UK.

e University of Santiago de Compostela, Galicia, Spain.

${ }^{f}$ University of Liverpool, Liverpool, UK.

$g$ University of Oxford, Oxford, UK.

E-mail: richard.plackett@cern.ch

\begin{abstract}
A series of tests in CERN's North Area beam facility have been used to demonstrate the suitability of the Timepix chip, combined with a silicon sensor, as a particle tracking device. Specifically of interest is the potential of a successor to the current chip to be used in the context of an $\mathrm{LHCb}$ VELO upgrade. The $55 \mu \mathrm{m}$ square pixels, large active fraction and analogue information make the chip very attractive for forward, high precision tracking systems such as the VELO.

In this contribution preliminary results are presented showing the resolution achieved by a Timepix assembly in a $120 \mathrm{GeV} \pi$ beam, over a wide range of incident angles. At the optimum angle the detector was able to provide an unbiased track residual of $5.5 \mu \mathrm{m}$. The telescope constructed for these measurements contributed a track extrapolation error of $2.5 \mu \mathrm{m}$. The plans for a future development of this telescope, also based on Timepix assemblies are discussed, with proposals for upgrading its spatial and timing resolution.
\end{abstract}

VERTEX 2009 (18th workshop) - VERTEX 2009

September 13 - 182009

Veluwe, the Netherlands

\footnotetext{
* Speaker.
} 


\section{Introduction}

A series of tests in charged particle beams were performed in order to demonstrate the potential of the Timepix[1] chip combined with a silicon sensor in a tracking system. Specifically the tests were aimed at evaluating the behaviour of the chip in the context of a future upgrade to the LHCb VELO[2]. The tests were carried out at the North Area beam lines at CERN's SPS facility between June and August 2009. Measurements were made of the resolution and efficiency of Timepix assemblies at different angles, with a range of sensor bias voltages and threshold levels.

\section{The Timepix Chip}

The Timepix readout chip is derived from the Medipix2[3] chip which was developed for single photon counting applications. The development of the Timepix chip took place within the context of the Medipix2 collaboration at the request of and with support from the EUDet Consortium[4].

The Timepix ASIC comprises a 256 by 256 matrix of $55 \mu \mathrm{m}$ square pixels each of which has its own analogue and digital circuitry. A globally applied shutter signal determines when all pixels are active. Each pixel contains a preamplifier, a discriminator with a globally adjustable threshold with 3-bit in pixel trimming, followed by mode control logic and a thirteen bit counter. It is possible to individually program the pixels to operate in different modes from their neighbours and be read out simultaneously. The three modes of operation are counting, time of arrival (ToA) and time over threshold (ToT). In counting mode the Timepix pixels behave in a very similar manner to the Medipix2 pixels, incrementing the counter each time the output of the amplifier passes the threshold set. As the Timepix only has one threshold per pixel the Medipix 2 charge windowing functionality is not available. In ToA mode the pixel records the time it was first hit. The counter is started when the amplifier first passes threshold and is stopped when the shutter closes. In this mode the depth of the counter limits the shutter opening, a particular limitation at high clock frequencies. Beyond 11,810 counts the counter overflows. In ToT mode the pixel measures the energy deposited during the time the shutter was open. The clock and counter are used to record the time the amplifier signal is above threshold. The amplifier has been designed so that this value is linear over a large charge range and can be tuned depending on the clock frequency and amplifier settings. The whole chip is read out after the shutter goes low with a dead time of $\sim 10 \mathrm{~ms}$. A clock signal, up to $100 \mathrm{MHz}$, is supplied externally to the chip and is propagated to each pixel on the matrix.

The Timepix and Medipix2 ASICs used for the telescope were bump bonded to $300 \mu \mathrm{m}$ thick planar silicon sensor chips which were nominally biased to 100V. Although the Timepix was designed primarily as an electron cloud detector without a sensor, it retains the Medipix 2 threshold equalisation and sensor polarity circuitry that make it equally well suited to silicon sensors.

In these tests the Timepix chips were predominantly used in ToT mode as it allowed accurate track position calculations to be performed when charge was deposited in multiple pixels by the same particle. This gave us the opportunity to reconstruct the position of the particle 'hit' with an accuracy better than the pixel's $55 \mu \mathrm{m}$ pitch.

As the Timepix and Medipix2 chips are read out using a shutter signal in a 'camera' like manner and there is no delay line or pipeline implemented in the pixels, it is not possible to use 
a trigger from a scintillator to read out a selected event. However, provided the hits are sparsely distributed across the chip several hundred tracks can be accumulated in one 'frame' whilst the sensor is continuously sensitive and then read out when the shutter is closed.

\section{LHCb VELO Pixel Upgrade}

$\mathrm{LHCb}[5]$ is a precision heavy flavour experiment that has just begun operation at the LHC at CERN. It is a forward angle single arm spectrometer with strong vertexing and particle identification capabilities, specifically targeted to B meson identification and reconstruction.

The $\mathrm{LHCb}$ detector is currently optimised to record events containing a single proton-proton interaction within an LHC bunch crossing. The trigger system[6] specifically rejects events involving multiple primary collisions and the LHC beam is weakly focused to maximise the number of events with single interactions. LHCb will see a luminosity of $2 \times 10^{32} \mathrm{~cm}^{-2} \mathrm{~s}^{-1}$ in these nominal conditions. To allow $\mathrm{LHCb}$ to gather statistics at a higher rate an upgrade to increase the luminosity by an order of magnitude is planned. However, it is not possible to optimise the current trigger system for a greater number of events as simulations indicate that in hadronic channels the triggers rapidly saturate and degrade. To overcome this limitation the detector will be completely read out at the $40 \mathrm{MHz}$ LHC bunch crossing frequency, with all the data processing happening on a CPU farm. This 40 fold increase in the readout rate requires the replacement of most of the front end electronics of the various sub-detectors.

The current VErtex LOcator (VELO)[7] sub-detector in LHCb is a double sided strip detector with an $r \phi$ geometry surrounding the interaction point. There are concerns that as the number of primary interactions increases the strip ambiguities will reach a level where they compromise the detectors performance. Therefore a pixel solution is being considered, despite the challenges of radiation tolerance, power consumption and increased material in such an environment. A pixel detector based on Timepix technology is a strong candidate for an upgraded VELO due to its square pixels and large active area allowing a thin module to be produced.

Up to this point neither Medipix2 nor Timepix had been operated as a perpendicular particle tracker, the measurements described here are intended to provide experience with the ToT technique and square pixel concept. Also, the efficiency and resolution that can be achieved with a current Timepix detector were measured. To ensure the detector response was comparable with a future LHC system all the tests were carried out in a $120 \mathrm{GeV}$ pion beam.

\section{Timepix Telescope}

It was initially foreseen that measurements of the Timepix assembly would be carried out in a pre-existing telescope. However, due to limited access as main user, the effort which would have been required to synchronise the two systems and the modest frame of the existing telescope, it was decided to construct a telescope using a further four Timepix and two Medipix planes. This allowed the easy integration of the Device Under Test (DUT), provided complete compatibility between the readout systems and enabled an event rate of $200 \mathrm{~Hz}$. The layout of the Telescope is shown in Figure 1. 


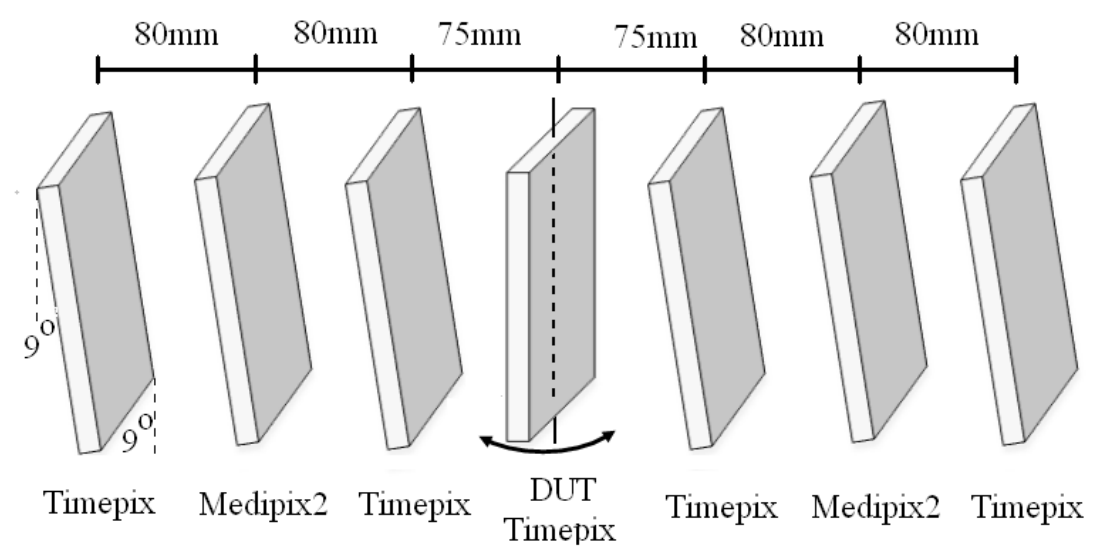

Figure 1: A diagram of the pixel detector assemblies within the telescope, showing the angled four Timepix and two Medipix2 detectors and the Timepix DUT with its axis of rotation. The separation of the devices shown is as measured from the canters of the chips and was enforced by the angle and the connection to the readout systems.

In order to maximise the spatial resolution achieved by the telescope, the reference detectors were fixed at $9^{\circ}$ to both the horizontal and vertical axes perpendicular to the beam line. A simple geometrical model of the pixel demonstrates that at this angle particles must traverse more than one pixel. The expected optimum angle is $\tan ^{-1}\left(\frac{\text { Pitch }}{\text { Thickness }}\right)$. This allowed the centroiding calculation incorporating the ToT information from the Timepix to be used for all nominal tracks. In addition the DUT was mounted at the centre of a symmetric arrangement of chips to further increase the resolution achieved. To allow a resolution measurement to be taken with the DUT at as many angles as possible, and to increase accuracy, the DUT was mounted on rotation and translation stages driven by stepper motors that allowed it to be turned and aligned remotely.

The Medipix2, and Timepix assemblies, including the DUT were read out using USB driven systems provided by CTU[8] and the Pixelman data acquisition and control software[9]. These are the standard, portable, low bandwidth readout systems used in most Medipix applications to date. Each USB unit is attached to one chip. A signal is applied simultaneously to all the USB readout units and its rising edge triggers local shutters to individual ships. The length of the shutter is programmable in each USB unit and it was set to be the same for each assembly. It was optimised on a run by run basis to capture between 100 and 500 tracks per frame depending on beam conditions. The microcontroller in the USB unit produces a delay between the trigger being received and the shutter being sent of $4 \pm 0.5 \mu \mathrm{s}$. As shutter periods of down to $10 \mathrm{~ms}$ were used depending on the beam intensity the error on the efficiency introduced by this jitter should be small. In situations where a shorter shutter is requires, such as high particle flux environments this will become a constraint on the use of the USB systems.

\section{Measurement Aims}

The main aim of the measurement campaign was to study the resolution and efficiency of the Timepix when combined with a planar silicon sensor and to directly compare this to an identical 
chip coupled to a double sided 3D silicon sensor[10]. The primary resolution measurement was the dependence of resolution with respect to sensor angle with the beam. Data points were taken between $\pm 18^{\circ}$ about the vertical axis, with the values about normal being sampled every degree. In addition data to determine resolution with respect to sensor bias, threshold level and the effect of ToT information was also recorded. From this data it will be possible to calculate the efficiency as a function of sensor bias, threshold, read out chip mode and angle. In addition a data set was recorded with the Timepix in ToA mode to investigate the effect of 'timewalk', where the pulse shape and discriminator reaction time affect the recorded arrival time. A subset of these measurements was repeated with the 3D Timepix to allow the direct comparison of the 3D and planar sensors.

\section{Telescope Alignment}

An initial gross alignment of the system to the beam was performed by locating the beam spot in the telescope sensors and manually adjusting the position of the mechanics. After this a rapid cluster symmetry analysis technique was used to determine the accurate alignment to the beam by scanning the DUT through its full range of movement. A numerical fit was made to the data and the zero angle determined to be at $-0.22^{\circ}$. It was decided that this level of misalignment of the system could be taken into account in the offline analysis, and that no significant loss of data would result. It was also found that imaging the beam spot with the Timepix and Medipix 2 detectors was an effective way of achieving a good initial alignment.

When the first data was recorded an initial hit correlation was performed to check that the telescope was operating correctly, that the planes were not grossly misaligned, and that the synchronisation was reasonably precise. This also provided information on the overlap of the telescope sensors with the DUT which was necessary to effectively estimate the number of tracks collected in each frame. The analysis software that was developed used a $\chi^{2}$ minimisation based on the Minuit[11] library to perform a final alignment of the sensor planes in the rotation and translation modes perpendicular to the beam. A similar minimisation of 'angled tracks' caused by scattering was used to get an accurate estimation of the rotation and translation modes parallel to the beam.

As it is difficult to accurately parameterise the deposition of charge in a specific silicon sensor, and nominal deposition is not best modelled by a simple centroiding algorithm, an ' $\eta$ correction'[12] was required to take full advantage of the resolution of the telescope. By interactively mapping the hit position reconstructed by the telescope to the position reconstructed from the cluster, this source of uncertainty was reduced. As can be seen in Figure 2 a truly linear mapping of the cluster can only be achieved in circumstances when the sensor is at a large enough angle to always leave a multi pixel cluster from any hit. This was ensured in the telescope by the inclination of the sensor planes.

\section{Preliminary Results}

Preliminary results from the analysis of the resolution are available. Figure 3 shows the resolution achieved at the DUT with a minimum of $5.5 \mu \mathrm{m}$ at the geometrically predicted position of $9.5^{\circ}$ which matches with predictions. Included in this is this the tracking uncertainty of the telescope at the DUT which was just $2.5 \mu \mathrm{m}$. It should be noted that this was achieved with the inclusion of 


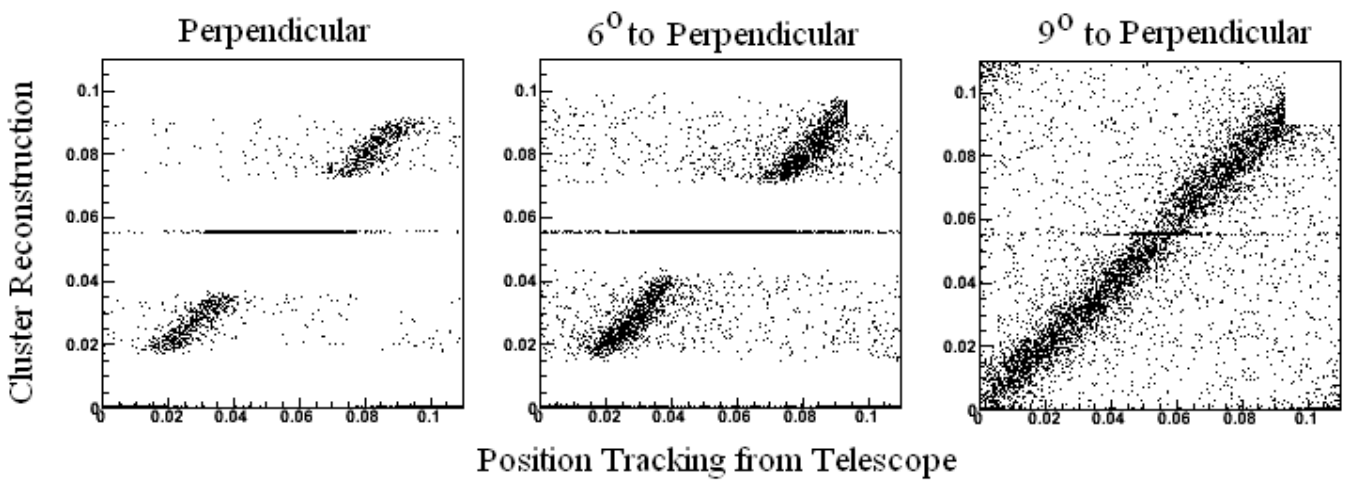

Figure 2: The correlation between the one dimensional sub-pixel position reconstructed by analysing the clustering after the $\eta$ correction and the position returned by the telescope tracking information. The linearity achieved by the $\eta$ correction can be clearly seen, as can the effect of single pixel clusters where the charge sharing information cannot be used.

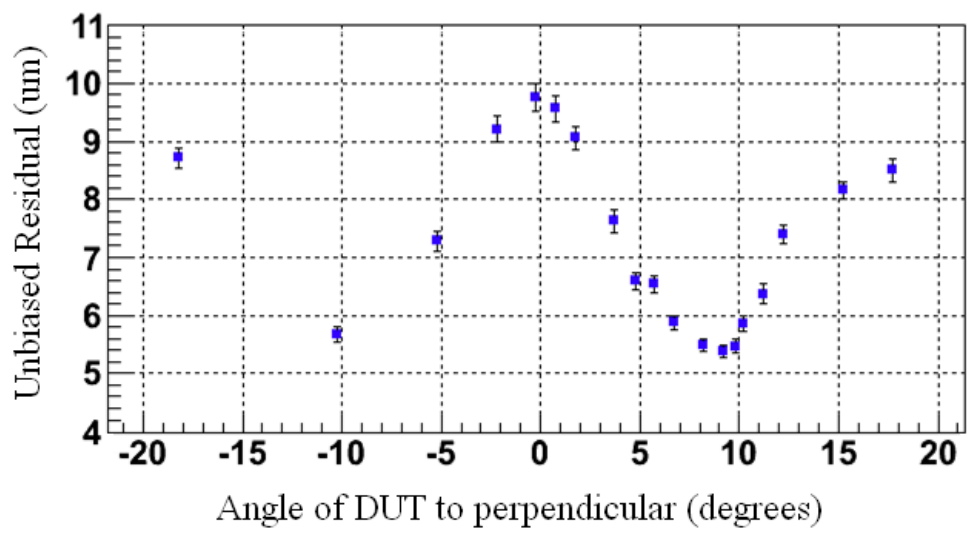

Figure 3: The resolution of the DUT plotted against its angle to perpendicular. This shows the best resolution at the expected position of 9.5 degrees, as well as confirming the expected resolution pattern is followed. Of this optimum resolution of $5.5 \mu \mathrm{m}, 2.5 \mu \mathrm{m}$ is a contribution, quadratically added, from the telescope itself.

all the reconstructed tracks and without a 'flat field' [13] gain correction to the DUT or telescope detectors. The resolution quoted for the DUT is the 'unbiased residual', the rms width of the distribution of offsets of the hit to the expected position of the track, without including the hit itself in the track generation. The uncertainty contribution given for the telescope is the 'track extrapolation error', the uncertainty on the track position projected onto the DUT plane.

By utilising the data from the telescope planes it has been possible to directly compare the resolution of a Timepix detector providing analogue ToT information with a Medipix2 detector providing pure binary information. As Figure 4 shows there is an improvement of the resolution, from $11 \mu \mathrm{m}$ to $5 \mu \mathrm{m}$ in the best case situation. This has implications for the design of the VELO pixel chip, and this analysis is being actively pursued.

The initial efficiency scan of the 3D sensor shows the inefficient regions, marked $\mathrm{a}$ and $\mathrm{b}$ 

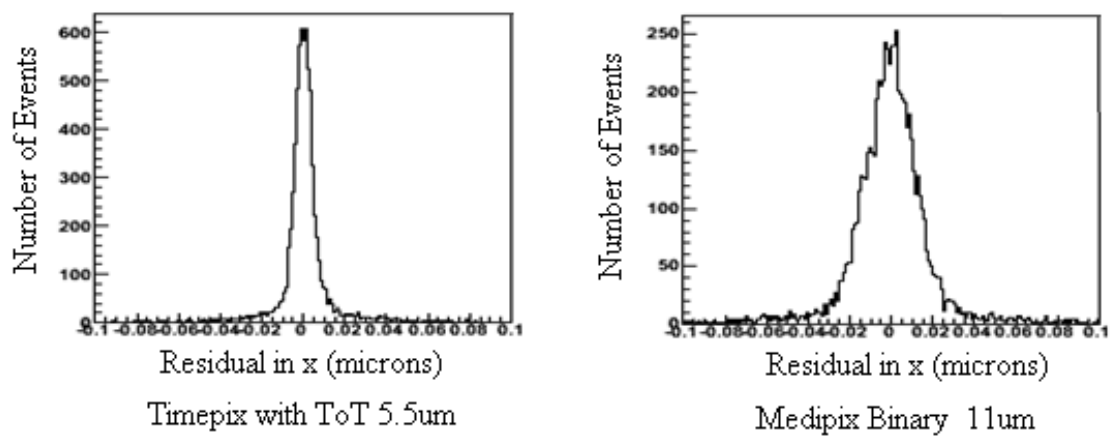

Figure 4: The residuals of the Timepix operating in ToT mode and the Medipix providing binary information are shown in these plots. The widths provide the resolutions of $5.5 \mu \mathrm{m}$ and $11 \mu \mathrm{m}$ respectively, demonstrating the improvement possible by making use of the analogue information from the pixels. As both of these sensors were in the telescope they were angled at $9^{\circ}$ to the

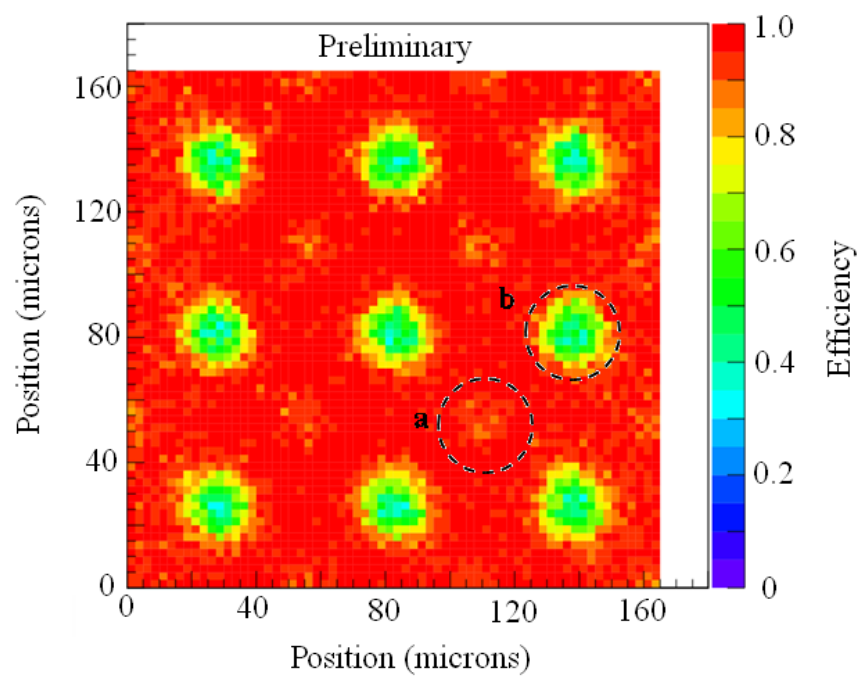

Figure 5: A map generated by overlaying 3 by 3 pixel segments of the matrix and plotting the average efficiency for hits falling into each pixel. The marked areas $\mathrm{a}$ and $\mathrm{b}$ are the images caused by the insensitive column structures passing through the silicon.

in Figure 5. These correspond to particles that pass through the insensitive columns within the structure of the sensor. The figure shown is a composite of efficiencies from across the detectors and displayed as a 3 by 3 pixel region.

In addition to these results analysis work is ongoing on the measurements described in Section 5 .

\section{Future Timepix Telescope}

A notable success during this measurement campaign was the performance of the telescope 
constructed for the task. The simplicity of operation, fast setup time and high resolution achieved have proved it to be a powerful and versatile piece of equipment. This has lead to plans to construct a successor, building on the strengths and eliminating some of the weaknesses.

It is planned to improve the spatial resolution of the telescope, currently $2.5 \mu \mathrm{m}$, by using only Timepix detectors as sensor planes, with four detectors per arm. The assemblies will be mounted on lower mass PCBs that will be cut away behind the sensor to reduce scattering. With these improvements it is hoped that the spatial uncertainty at the DUT can be reduced to less than $2 \mu \mathrm{m}$.

The major limitation of the current telescope is its slow readout rate recording on average only one frame of up to 500 tracks per second during a beam spill. This is due to the use of the USB1 interface and a very simple synchronisation scheme. It uses only a small fraction of the potential bandwidth available from the Timepix chips. By next year two new readout systems should be available, RELAXD[14] being developed by NIKHEF and the USB2 system[15] under development at CTU. With either of these it should be possible to increase the frame rate of the system up to $150 \mathrm{~Hz}$, giving a maximum track rate of on the order of $75 \mathrm{kHz}$ during a beam spill.

Although both of these systems should have a significantly smaller shutter opening jitter than the USB1 system, a fast offline synchronisation system is highly desirable to provide accurate timing. This is required to test sLHC systems in asynchronous beams without being subject to effects caused by out of time particles. By using a FPGA based timing system to record shutter and scintillator signals it should be possible to record track timings to the order of $1 \mathrm{~ns}$. By linking this to a single Timepix plane in each arm operating in ToA mode, it will be possible to assign these precise timings to individual tracks.

\section{Conclusions}

The preliminary resolution measurements presented here show that the Timepix concept is a good candidate for the LHCb VELO upgrade. The requirement to make full use of analogue ToT information to improve the resolution is demonstrated by the comparison with the Medipix 2 assemblies. The measured Timepix resolution is $5.5 \mu \mathrm{m}$ at the optimum angle of $9.5^{\circ}$ in agreement with geometrical predictions. The telescope constructed for these measurements, comprising Timepix and Medipix 2 detectors achieved a resolution of $2.5 \mu \mathrm{m}$. The analysis effort is ongoing and further results from the measurements made will be available soon. These will include detailed efficiency and timing measurements as well as comparisons of binary and analogue data at different angles.

The success of the Timepix Telescope used in these tests has prompted plans for further development of a system based on these detectors. It is hoped that a spatial resolution of better than $2 \mu \mathrm{m}$ and a time resolution of $1 \mathrm{~ns}$ can be achieved. The upgraded telescope will be able to run at a rate of $\sim 75,000$ tracks per second.

\section{Acknowledgements}

The authors would like to thank the CMS SiBit and EUDET telescope teams as well as the LCFI/Spider and SILC/Fortis collaborations for the use of their beam time and facilities. Without their help and support this work would not have been possible. Thanks are also due to the Medipix group at CTU Prague who provided the USB readout electronics. In addition the CERN 
Marie Curie ACEOLE scheme provided funding to allow much of this work to be carried out and presented.

\section{References}

[1] X. Llopart et.al.,'Timepix, a 65k programmable pixel readout chip for arrival time, energy and/or photon counting measurements', NIM A 581 (2007) 485-494.

[2] The LHCb Collaboration, 'Expression of Interest for an LHCb Upgrade', CERN LHCC 2008-007.

[3] X. Llopart et.al., 'Medipix2: a 64k Pixel Readout Chip With 55 4 m Square Elements Working in Single Photon Counting Mode', IEEE Trans. Nucl. Science Vol.49, No.5, Oct 2002.

[4] http: //www.eudet.org/

[5] The LHCb Collaboration, 'The LHCb Detector at the LHC', 2008 JINST 3 S08005.

[6] The LHCb Collaboration, 'LHCb Trigger System', CERN LHCC 2003-031.

[7] The LHCb Collaboration, 'LHCb Vertex Locator', CERN LHCC 2001-011.

[8] J. Jakubek, Z. Vykydal, S. Pospísil, 'USB Interface for Medipix2 Pixel Device Enabling Energy and Position Detection of Heavy Charged Particles', NIM A 563 (2008) 112-115.

[9] T. Holy, J. Jakubek, S. Pospísil, J. Uher, D. Vavrík, Z. Vykydal, 'Data acquisition and processing software package for Medipix-2 device’, NIM A 563 (2006) 254-258

[10] D. Pennicard, G. Pellegrini, M. Lozano, C. Fleta, R. Bates, C. Parkes, 'Design, simulation, production and initial characterisation of 3D silicon detectors', NIM A 598 (2009) 67-70.

[11] http://lcgapp.cern.ch/project/cls/work-packages/mathlibs/minuit/ index.html

[12] R. Turchetta, 'Spatial Resolution of Silicon Microstrip Detectors', NIM A 335 (1993) 44-58.

[13] flat field gain correction

[14] J. Visschers, S. Deniz, K. De Munck, T. Borgers, W. Ruythooren, P. De Moor, Z. Vykydal, 'The RELAXd project: development of four-side tilable photon counting imagers', NIM A 591 (2008) 241-244.

[15] M. Platkevic, V. Bocarov, J. Jakubek, S. Pospísil, Z. Vykydal, 'Signal processor controlled USB2.0 interface for Medipix2 detector', NIM A 591 (2008) 245-247. 